\title{
Community Pharmacy Prescription Screening in the UAE
}

\author{
Muaed Alomar
}

Al Ain University, The United Arab Emirates.

Email: muayyad74@yahoo.com

Received November $24^{\text {th }}, 2013$; revised December $25^{\text {th }}$, 2013; accepted January $6^{\text {th }}, 2014$

Copyright (C 2014 Muaed Alomar. This is an open access article distributed under the Creative Commons Attribution License, which permits unrestricted use, distribution, and reproduction in any medium, provided the original work is properly cited. In accordance of the Creative Commons Attribution License all Copyrights (C) 2014 are reserved for SCIRP and the owner of the intellectual property Muaed Alomar. All Copyright (c) 2014 are guarded by law and by SCIRP as a guardian.

\section{ABSTRACT}

This study was designed to investigate and to evaluate pharmacists in the United Arab Emirates-Fujairah-DibbaSharjah-Abu Dhabi. The study is concerned with how pharmacists deal with prescriptions (OTC-Ethical) and their behavior with their patients in comparison to standards. Objectives: The study's objective is to evaluate current pharmacists, who deal with a large number of patients and affect their lives. Methodology: A descriptive and comparative prospective prescription screening study, which evaluates data in OTC and ethical prescriptions.1000 prescriptions (including OTC and ethical) were collected randomly from community and hospital pharmacies (Fujairah, Dibba, Sharjah and Abu-Dhabi) in the United Arab Emirates. Data collection was carried out within a 3-month time period. SPSS (version 16) was used for data entry and analysis in which each prescription was evaluated using certain parameters. Results: One thousand prescriptions were collected, of which $\mathbf{1 0 . 6 \%}$ were OTC and $\mathbf{8 9 . 4 \%}$ were ethical, which were included in the analysis. Analysis was carried out to obtain percentages, frequencies and general cross tabs. Conclusion: Our study showed that most patients received no oral or written information about how to use the medication, how to manage adverse effects, what precautions to take, or what to do in case of an overdose. Also, some patients were not asked basic questions by the pharmacist that were needed to assess them, which is very far from the concept of pharmaceutical care delivered all over the world.

\section{KEYWORDS}

\section{Prescription; Screening; Errors; Medications}

\section{Introduction}

The roles and responsibilities of pharmacists in delivering healthcare have changed drastically over the years. Starting at the beginning of the 20th century, the pharmacist's main duties were to prepare and deliver appropriate medicinal products to patients. At that time, pharmacists served as a trusted source of health information and patient advice for the surrounding communities. During the middle of the 20th century, pharmaceutical industries started to take over the pharmaceutical preparation and formulation [1].

The nineteen sixties and seventies is considered the time when pharmacists became more involved in clinical settings and in direct contact with patients, nurses and physicians. The responsibilities have moved from formulation to patient counseling and education.
Health professionals must work to safeguard patients against preventable, adverse effects associated with potent and potentially dangerous chemical agents.

While pharmacists have maintained the responsibility for the safe preparation and distribution of medications, the pharmaceutical care definition of pharmacy practice has moved the pharmacist's role into the domain of collaborative direct patient care, with that domain's incumbent responsibilities for assessing patients' status, developing and implementing a therapeutic plan, documentation and follow-up of outcomes, and assuming responsibility for those outcomes.

Conversely, physicians often lack important information and knowledge that pharmacists possess, such as the patient's total medication profile and compliance with refill orders and drug information [2]. 
Teamwork should provide substantial improvement in health outcomes for ambulatory patients, especially those with chronic diseases and those who see multiple physicians but receive care from a single pharmacist [1].

A prescription is a way of communication between the prescriber and the dispenser; it is also a pharmaceutical care program utilized by a physician or other qualified practitioner in the form of instructions in order to implement a certain pharmacotherapeutic plan [3].

\section{Contents of prescription}

Belknap and Lanzotti state that any prescription should contain the following information [2]:

A) The name and address of the patient.

B) The name and quantity of the drug or device prescribed and the directions for use.

C) The date of issue.

D) Medical condition.

E) Date of issuance.

F) The original signature of the prescriber.

G) The prescriber's name and business address.

$\mathrm{H})$ Room number and route of administration if the patient is hospitalized.

I) Number of refills allowed.

\section{Prescription Error}

Medication errors are very common in the medical field; they are divided into two types: those detected during patient counseling, and those reported after patients left the pharmacy [4]. Dispensing errors are a discrepancy between the prescriber's interpretable written order and the filled prescription [5]. For refill prescriptions, medication errors are defined as any deviation between the information of the filled prescription and the contents described on the pharmacy-generated prescription label [6].

Prescription errors are very common, ranging from harmless to potentially dangerous and life threatening [7]. Potential medication errors, which most likely to happen during ordering and administration of the prescription, are preventable. Prescribing errors encompass acts of commission (giving drugs that are contraindicated or unsuitable) and acts of omission (failure to prescribe drugs when indicated) [8]. Prescription errors in the USA range from $0.2 \%$ to $10 \%$. Using conservative estimates of a $1 \%$ dispensing error rate and an annual total of 3 billion dispensed prescriptions, a projected 30 million errors would occur each year in United States [6]. Dean, et al. and Abdullah, et al. elaborated that most of the errors (54\%) were associated with choice of dose $[9,10]$. While the majority of all errors (61\%) originated in medication order writing, most serious errors (58\%) originated in the prescribing decision [7].

Pharmacists have the ability and motive to implement pharmaceutical care in order to improve therapeutic out- comes and patients' quality of life using the available resources; patient counseling is an integral part of the pharmaceutical care model [11]. The patient is the pharmacist's main source of information. Dialogue and discussion between pharmacist and patient help pharmacists uncover the needs of the patient and manage therapeutic plans in the best manner [12].

Henk, et al. found that on average one in 200 prescriptions $(0.49 \%)$ was found to have been positively modified by Dutch community pharmacists [13]. About half of these interventions (49.8\%) were aimed at preventing adverse drug reactions; $29.2 \%$ were rated as a positive modification in the effectiveness of pharmacotherapy and 8.6\% affected both effectiveness and adverse drug reaction. By extrapolating their data, they estimated a daily occurrence of approximately 2700 positive interventions in all Dutch pharmacies (1.6 per pharmacy per day) [13].

\section{Methodology}

\subsection{Objectives}

The objective of this study is to evaluate and compare physician's prescriptions in the community and hospital pharmacies, and to evaluate the lack of information provided by community pharmacists regarding OTC prescriptions.

\subsection{Type of Study}

A descriptive and comparative, prospective prescription screening study, which evaluates data in OTC and ethical prescriptions.

\subsection{Sampling Technique}

A simple random sampling technique was used. 1000 prescriptions (including OTC and ethical) were collected randomly from community and hospital pharmacies.

For OTC prescriptions, the researcher would attend and listen to, record and analyze the conversations between patients and pharmacists. For ethical prescriptions, the researcher would collect prescriptions, watch the conversation if there was any, and collect information.

Areas included in the sampling were Fujairah, Dibba, Sharja and Abu-Dhabi in the United Arab Emirates.

\section{Data Analysis}

SPSS (version 16) was used for data entry and analysis in which each prescription was evaluated using the following variables:

NO: Prescription number. This is the serial number given to each prescription for further reference.

TOP: Type of prescription. The answer is one of the two previously defined choices: OTC or ethical

Date: Is there date specified on the prescription? The 
answer is one of the three previously defined choices: yes, no or not applicable.

Gender: Sex of the patient. The answer is one of the three previously defined choices: male, female or not mentioned.

Age: Determines if age is mentioned or not. The answer is one of the two previously defined choices: mentioned or not mentioned.

Weight: Determines if weight is mentioned or not. The answer is one of the two previously defined choices: mentioned or not mentioned.

Martial: If the patient is married, single, divorced, widow; choices: single, married, divorced, widow or not mentioned.

Writing error: In case of ethical prescription, were there any writing errors; in case of OTC prescription, writing error is not applicable; the answer is one of the three previously defined choices: yes, no or not applicable.

Missing: In case of ethical, was there any missing data (dosage form, product strength, quantity to be dispensed)? Choices given are: yes (dosage form), yes (product strength) yes (quantity to be dispensed), no, not applicable.

Contraceptives: Is the patient taking contraceptives. The answer is one of the four previously defined choices: yes, no, pharmacist did not ask or not applicable.

Pregnancy: If pregnant or not. The answer is one of the four previously defined choices: pregnant, not pregnant, not known or not applicable.

Trimester: If pregnant, then which trimester. The answer is one of the four previously defined choices: specified, not specified or not applicable.

Feeding: If breast feeding or not. The answer is one of the four previously defined choices: breast feeding, not breast feeding, not applicable or not specified.

OPDS: If there is any other disease other than the current medical problems. The answer is one of the two previously defined choices: specified or not specified.

OPD1: If there is any other disease other than the current medical problems then what are they? Choices listed in the system include: Cardiovascular, Respiratory, Gastrointestinal tract, Cerebral, Skin, Muscular, Skeletal, Hair, Sight, Sexual, Bacterial infection, Viral infection, Fungal infection, Cancer, Others, Non specified.

Allergy: Is the patient allergic to any kind of food or medication? The answer is one of the five previously defined choices: allergic to food, allergic to antibiotic, not known, pharmacist did not ask or not applicable in case of ethical prescription.

FH: In case of OTC prescription did the pharmacist ask the patient about disease family history? The answer is one of the three previously defined choices: yes, no or no need.
PMH: past medication history (medication used chronically). Did the patient take any other medications? The answer is one of the four previously defined choices: yes, no, pharmacist did not ask or not applicable.

NSAIDS: If the patient is taking Non Steroidal Anti-inflammatory Drugs or not. The answer is one of the three previously defined choices: yes, no or pharmacist did not ask.

Antibiotic: If the patient is taking antibiotics or not. The answer is one of the three previously defined choices: yes, no or pharmacist did not ask.

Antihypertensive: If the patient is taking anti hypertensives or not. The answer is one of the three previously defined choices: yes, no or pharmacist did not ask.

Antidepressant: If the patient is taking antidepressants or not. The answer is one of the three previously defined choices: yes, no or pharmacist did not ask.

Hypoglycemic: If the patient is taking hypoglycemic medications or not. The answer is one of the three previously defined choices: yes, no or pharmacist did not ask.

Symptoms: In case of an OTC prescription, did the pharmacist ask about symptoms? The answer is one of the three previously defined choices: yes, no or not applicable in case of ethical prescription.

Onset: In case of an OTC prescription, did the pharmacist ask about onset? The answer is one of the three previously defined choices: yes, no or not applicable in case of ethical prescription.

Annoyed: In case of an OTC prescription, did the pharmacist ask about if the disease gets worse by any kind of action? The answer is one of the three previously defined choices: yes, no or not applicable in case of ethical prescription.

Incidence: In case of an OTC prescription, was there any question regarding the frequency of the disease? The answer is one of the three previously defined choices: yes, no or not applicable in case of ethical prescription.

Smoker: In case of an OTC prescription, was there any question regarding smoking? The answer is one of the three previously defined choices: yes, no or not applicable in case of ethical prescription and infant.

Alcohol: In case of an OTC prescription, was there any question regarding alcohol consumption? The answer is one of the three previously defined choices: yes, no or not applicable in case of ethical prescription and infant.

Financial status (abbreviated in the system as poor): Did the pharmacist ask if the patient can afford the treatment or not? The answer is one of the two previously defined choices: yes or no.

TMS: Did the pharmacist tell the patient how to take the medication? The answer is one of the three previously defined choices: yes (dose) or no, yes (dose + recom- 
mendation).

Monitoring: Was there any need for monitoring? If there is a need, was there any reaction from the pharmacist? The answer is one of the three previously defined choices: yes, no or no need.

Dose: Was there any counseling about missing doses? The answer is one of the two previously defined choices: yes or no.

Food: Was there any counseling regarding medication use and food intake? The answer is one of the two previously defined choices: yes or no.

Overdose: Did the pharmacist advise the patient about the symptoms of overdose and toxicity and how to deal with it? The answer is one of the two previously defined choices: yes or no.

Call me: Did the pharmacist offer his telephone number for further enquiry from the patient? The answer is one of the two previously defined choices: yes or no.

Himself: Did the pharmacist deal directly with the patient or somebody on behalf of the patient? The answer is one of the two previously defined choices: patient himself or somebody else.

Referral: Did this patient's condition require referral? The answer is one of the three previously defined choices: yes, no or not applicable in case of ethical prescription.

Referred: If the patient's condition requires referral, was he referred to the specialist or hospital? The answer is one of the three previously defined choices: yes, no or not applicable in case of ethical prescription.

Interaction: Were there any drug-drug interactions either between the newly prescribed medications or ones already taken? The answer is one of the three previously defined choices: yes for newly prescribed, yes for all drugs, no for newly prescribed or no for all.

ADR: Did the pharmacist deal with medication side effects or adverse reaction? The answer is one of the two previously defined choices: yes or no.

\section{Results}

One thousand prescriptions were collected, of which $10.6 \%$ were OTC and $89.4 \%$ were ethical; $0.4 \%$ of collected data are not specified on the prescriptions.

The percentage of missing data in ethical prescriptions (dosage form, product strength, quantity to be dispensed) was $4.8 \%$. About $0.4 \%$ of ethical prescriptions contain writing errors. By not providing full details about sex, age, weight, status (married or single), this can lead to confusing data, e.g. $3 \%$ of prescriptions did not mention the sex of the patient; $14.5 \%$ of prescriptions did not mention the age of the patients; $98 \%$ did not mention the weights of the patients, in $95.5 \%$ of prescriptions the status of the patients was not mentioned. In $99.2 \%$ of prescriptions, the pharmacist did not ask about the past medication history (medications that are used chronical- ly). Approximately $30.4 \%$ of patients take NSAIDs and $36.8 \%$ of pharmacists did not ask if NSAIDs were taken or not. $24.1 \%$ of patients took antibiotics and $38.6 \%$ of pharmacists did not ask if they were taken or not. $25.1 \%$ of patients took antihypertensive drugs and $39 \%$ of pharmacists did not ask if they were taken or not. $1.8 \%$ of patients took antidepressants and about $49.5 \%$ of pharmacists did not ask if they were taken or not. 12.8\% of patients took hypoglycemic drugs and $45.2 \%$ of pharmacists did not ask if they were taken or not. There were $0.3 \%$ of female patients taking contraceptives and $48.7 \%$ of pharmacists did not ask any questions regarding contraceptives. Approximately $1.1 \%$ of female patients were pregnant; of these patients, $0.1 \%$ it was specified which trimester they were in and $48.4 \%$ were not; $48.2 \%$ of female patients were not asked by the pharmacist about their trimester. Approximately $0.1 \%$ of female patients were breast feeding and $26.3 \%$ were not specified. Approximately $0.4 \%$ of patients specified that they had diseases other than the current medical problems e.g.: $0.2 \%$ had cardiovascular problems, $49.9 \%$ had other problems and about $49.8 \%$ were not specified. Approximately $0.1 \%$ of patients were allergic to antibiotics and $99.8 \%$ of patients were not asked by the pharmacist about antibiotic allergies.

\section{OTC Prescriptions}

In the case of OTC prescriptions, approximately 55.9\% of pharmacists did not ask about the patient's family history.

Furthermore, in the OTC prescriptions, only $4.9 \%$ of the patients were asked by the pharmacist about symptoms, $52.5 \%$ did not ask and $42.5 \%$ was not applicable. Approximately $1.5 \%$ of pharmacists asked the patient about the onset of disease, $55.8 \%$ the pharmacist did not ask and about $42.6 \%$ was not applicable. Approximately $0.7 \%$ of pharmacists asked if the disease was exacerbated by any kind of action, $56.1 \%$ the pharmacist did not ask and $43.1 \%$ was not applicable. Approximately $0.8 \%$ of pharmacists asked if the disease occurred frequently or not, $56 \%$ the pharmacist did not ask and $43.1 \%$ was not applicable. $1.2 \%$ of patients were smokers, $43.3 \%$ was not applicable and $55.4 \%$ of patients were not asked by the pharmacist if they were smokers. $4.7 \%$ of patients consumed alcohol, $43.4 \%$ were not applicable and in $51.8 \%$ of cases the pharmacist did not ask the patient about alcohol consumption. $73.9 \%$ of pharmacists did not tell the patient how to take the medication, and $18.4 \%$ of pharmacists only informed patients about the dose; however, $2.8 \%$ of pharmacists informed the patient about the dose and recommendations. Approximately $45.4 \%$ of pharmacists did not ask the patient if he or she could afford the treatment or not and $51 \%$ of pharmacists asked patients if the treatment could be afforded. Pharmacists 
carried out a dose regimen for $29.2 \%$ of patients; $25.1 \%$ of pharmacists did not carry out a dose regimen and $40.8 \%$ did not require a dose regimen. About $94.7 \%$ of pharmacists did not inform the patient what to do if he or she missed the dose with only $0.4 \%$ of pharmacists informing patients what to do in the case of a missed dose.

According to the results $39.2 \%$ of patients needed referral, of which:

- $38.5 \%$ of patients were referred to hospital or a specialist whereas 50.6\% were not referred, and in 10.9\% of cases, referral was not applicable.

- $58.5 \%$ of patients did not need referral and $2.3 \%$ of cases were not applicable.

Approximately $1.9 \%$ of pharmacists dealt with medication side effects or adverse reactions and approximately $48 \%$ did not.

Of the prescriptions collected, 5.4\% drug-drug interactions for newly prescribed drugs, and $44.6 \%$ had no drug-drug interaction for newly prescribed drugs.

Approximately $94.9 \%$ of pharmacists did not offer a telephone number for further enquiry from the patient. $4.7 \%$ of patients do not take their medication by themselves.

Approximately 56.2\% of pharmacists did not advise the patient about the symptoms of overdose and toxicity and how to deal with it.

Approximately 93.1\% of pharmacists did not inform the patient about food and medication; Patients were not informed whether the medication should be taken before, after or with food.

\section{Discussion}

In routine medical settings, patients are generally not fully informed of the risks and benefits of each and every treatment they receive with. This results in $30 \%$ to $50 \%$ of patients not using medication as prescribed because they lack information [14,15].

Whenever pharmacists ask a patient what they want to know about their medications, patients say "everything", but that's not exactly right. What they really want to know is what their choices are. Patients also want to know how to manage probable and possible problems [15].

Patients believe that verbal counseling from pharmacists is the most effective way to obtain information about medications. Their most common complaints about counseling are lack of pharmacist interaction, lack of instruction, and inadequate explanation of printed materials. While patients felt that individual contact with pharmacists was important, about half reported having very little contact. Women were much more satisfied than men with the quantity and quality of their interactions with pharmacists. Patients perceived that technicians dispensed most prescriptions. The most negative comment was,
"When I first got my prescription, [the pharmacist] did not explain a damn thing to me. I did not even know what the medicine was for" [16].

Of almost universal concern to patients is the effect of the medication on sleep and activity. Patients want to know whether a medication will make them drowsy, cause them to gain weight, alter their libido, or make them anxious. Pharmacists can use the standard counseling session to determine what the patient needs and wants to know. They can also work with other members of the healthcare team to supplement discipline-specific teaching. In the end, however, they must respect the patient's inalienable right to choose, even if the choice is to forgo treatment [15]. The concerns are similar when explaining potential treatment risks. Common adverse effects might be mentioned, but rarer ones might not. What does this particular patient want to know? What does he or she consider important? The pharmacist's dilemma arises from wanting to provide the patient with the information he or she needs to optimize outcomes without needlessly alarming the patient [15].

In a market-driven healthcare economy, three principal values exist: managing and, if possible, lowering costs; increasing patient satisfaction; and improving the quality of patient outcomes. These values are consistent with efforts to achieve more integration of services and collaboration among providers [17].

The role of the public health pharmacist continues to be defined. The provision of public health pharmacy service is commonplace today. Any person can enter a community pharmacy to seek drug information and obtain assistance in selecting nonprescription medicines to care for common ailments. Any institutional healthcare worker can request pharmacist consultation for therapeutic drug monitoring or drug information. Few healthcare professionals routinely offer free services to the public as do pharmacists. Pharmacists have been providing public health services for decades and with greater frequency at present during the pharmaceutical era paradigm [18]. The percentage of patients who take several drugs for chronic diseases will continue to increase. Based on current trends, the number of patients who lack adequate access to care, or who receive either suboptimal, inappropriate, or unnecessarily expensive drug therapy for their acute and chronic diseases will increase. Even as financial and human resources are increasingly strained within the current health care system, costs will continue to rise unless changes are made [17]. Behaviors such as offering advice, information, and instruction and providing feedback on behaviors, thoughts, and feelings are a form of social support known as directive guidance. Directive guidance behaviors are among the most influential components of social support in increasing medication adherence [19]. Many of the activities involved in pharma- 
ceutical care such as providing patients with information about the proper use of medications, instructing them on how to deal with adverse effects, encouraging them to take their medications appropriately, and giving them feedback on how they are doing and how they can improve are forms of directive guidance and are essential to helping patients achieve the desired therapeutic outcomes [19]. It is not feasible to validate the accuracy of the extent of community pharmacist-conducted medication communication with patients in this study relative to actual behavior. This level of communication was much lower than the ideal [20]. Reasons given about this lack of communication is that patients may be uninterested; the pharmacist lacks time, language barriers and a lack of experience of pharmacist to communicate [20]. The present study is the first study in the UAE investigating and evaluating pharmacists' work. Our database included 1000 prescription in 4 states in the UAE of which $10.6 \%$ were OTC and $89.4 \%$ were ethical; $43.9 \%$ of males and $53 \%$ females. This study produced several important findings according to certain parameters. These findings are consistent with recent observational studies, which suggest that a considerable number of patients did not receive any oral information or reinforcement about the prescribed prescriptions. The majority of patients received no oral advice or reinforcement about how to use the medication, how to manage adverse effect or precautions, or what to do in the case of an overdose.

Kripalani, et al. proved that age, sex, insurance type, severity of illness, number of medications, and certain medication types were independently associated with prescription-related issues after discharge, are medication errors which may have a significant clinical and economic impact [21]. Pharmacists will consider 1 of 3 recommendations during each encounter depending upon the severity of symptoms being presented, underlying conditions, previous attempts at treatment on the part of the patient [22]. In our study, $14.5 \%, 98 \%, 95.5 \%$ of patient's age, weight, and martial status respectively were not mentioned. Pharmacists also failed to inquire about past medication history (the medications that used chronically) in $99.2 \%$ of prescriptions and $49.8 \%$ of patients were not specified to have another disease other than their current medical problems or not. In OTC prescriptions $52.5 \%$ of pharmacists did not ask the patient about symptoms, $56.1 \%$ did not ask about action worsening the disease, $56 \%$ did not ask about the frequency of the disease and $55.8 \%$ did not ask about the onset of the disease. Therefore, we predicted that the patient was not well assessed and this may result in significant medication errors according to studies. Pharmacists' involvement in hypertension management programs has been shown to improve blood pressure, goal attainment rates, patient knowledge of his or her condition, and adherence to treatment, as well as reduce drug interactions and costs. Pharmacists can play a pivotal role in bridging the communication chasm that exists between physicians and patients. Because of their close interaction with patients, pharmacists have an opportunity to reiterate messages about the contribution of hypertension to the macrovascular and microvascular complications of diabetes, the detrimental long-term effects of untreated hypertension or poorly controlled blood pressure, the importance of adherence to lifestyle modifications and therapeutic regimens, and the need for regular checkups. In this study, $25.1 \%$ and $12.8 \%$ of patients (who were asked by the pharmacist) were taking antihypertensive drugs and hypoglycemic drugs respectively; whereas, about $73.9 \%$ the pharmacist did not tell the patient how to take the medication. $18.4 \%$ of the pharmacists only informed the patient about the dose and $2.8 \%$ the pharmacist informed the patient about the dose and recommendations. Also, $25.1 \%$ of patients, did not receive a dose regimen when it was needed; approximately $94.7 \%$ of pharmacists did not tell the patient what to do if he or she miss the dose; $56.2 \%$ of pharmacists did not advise the patient about the symptoms or overdose and toxicity and how to deal with them and about $93.1 \%$ of pharmacists did not tell the patient about food and medication or whether medication should be taken before, after or with food. Findings demonstrate that pharmacists provided effective cognitive services and refute the idea that pharmacists must be certified diabetes educators to help patients with diabetes improve clinical outcomes [23].

Gaffney carried out a single-center study performed to evaluate the effectiveness of a comprehensive pharmacy care program including patient education and an adherence aid [24]. The six-month, prospective study consisted of pharmacist drug counseling, provision of an adherence aid, and regular follow-up visits. After 6 months, drug adherence increased to $96.9 \%$; this was associated with significant improvements in systolic blood pressure (from 133.2 to $129.0 \mathrm{~mm} \mathrm{Hg}, \mathrm{p}=0.02$ ) but not in diastolic blood pressure.

Therefore, we can conclude that especially those diseases that cannot be cured by drug therapy, lifelong follow up and patient education are needed to promote adherence to diet and medications and at every step along continuum from development of risk factors through the various stages of the disease, numerous opportunities exist for pharmacists to use their expertise to delay progression and improve patient outcomes [25]. Moving from the traditional role as medication prescriber and dispenser to more patient oriented activities, termed pharmaceutical care, is growing at a modest rate and has not been fully implemented in daily practice; although there is a clear increase in the number of studies pub- 
lished on the effect of pharmacist patient communication [26,27].

We also found that $48 \%$ of pharmacists did not tell the patient how to deal with side effects or adverse reactions and this was found to be critical. As some studies done in USA documented that a wide variety of drug therapy problems and causes, including adverse drug reactions (20.1\% of care plans), need for additional drug therapy (18.9\%), lack of patient adherence to therapy (16.3\%), incorrect medication being prescribed (14.1\%), and drug dose too high (10.0\%) [28]. It has also been stated that hospitals without pharmacist-provided ADR management had a $34.90 \%$ increase of ADRs, where the effects on healthcare outcomes are more dramatic [29].

It is also recommend to ask whether the patient is pregnant or not to allow the pharmacist to decide if treating a condition is worth the known or unknown risks of using a medication during pregnancy [30]. However, in this study we found that $48.2 \%$ of female patient were not asked whether they are pregnant or not and $26 \%$ of female patients were not asked whether they are breast feeding or not. This may result in risky decisions for those patients, their neonates or infants.

Recently, the clinical importance of food-drug interactions and herb-drug interactions has received increased attention (i.e. drug interactions are a documented source of mortality and morbidity [29]. There are many known alcohol-drug interactions, association between alcohol use and many pharmacologically treated disorders suggest that substantial numbers of patients may be at risk for alcohol-drug interactions. For example warfarin is more likely to elicit hemorrhage in patients who consume alcohol [29]. The use of tobacco was documented to produce substantial health-related economic costs to society due to its complications and effect on other drugs and is the single most common cause of preventable death and disease in the United States [31]. We observed that $55.4 \%$ of pharmacists did not ask if the patient was a smoker, $51.8 \%$ did not ask if the patient consumed alcohol and $93.1 \%$ did not tell the patient about food and medication, i.e. whether the medication should be taken before, after or with food. This indicates the carelessness of pharmacists about important points which may result in many pharmacological disorders and drug therapy problems. In addition, we observed that $45.4 \%$ of pharmacists consider the financial status of the patient which according to studies stated that low-income individuals may lack health insurance either because they are unemployed, their employer does not offer it, or they cannot afford it inhibit the patient's ability to access needed medical services [22]. For example, a patient may have Medicare as their primary insurance that covers medical and laboratory fees but may have limited prescription coverage; therefore, the patient will pay an additional out-of-network fee. This will result in lack of cognitive services including patient education, anticoagulation monitoring, asthma management services, and cardiovascular risk reduction services. Private third-party payers may or may not choose to accept pharmacists as providers for purposes of compensation for cognitive services.

Therefore, pharmacists should carefully set fees and provide patient-friendly payment options, and be clear and straightforward regarding pricing policies. On the other hand, insurance representatives should have a clear understanding that the pharmacist is seeking credentialing (process by which the insurance company approves a specific health care professional to be a provider for their clients and thus receive compensation for providing these services) on the medical benefits side of the company and not for prescription benefits [22].

About $94.9 \%$ of pharmacists did not offer a telephone number for further enquiry from the patient which does not match with recent standards. Brown stated that telephone follow-up by a community pharmacist proved to be an excellent opportunity for detecting and managing DRPs (Drug related problems) [29]. Also, Kripalani et al. stated that close follow-up of patients by telephone may also be a helpful approach to promptly identifying prescription-related issues and other problems so that providers can intervene before more serious complications arise [21].

$\mathrm{Wu}$, et al. stated that telephone counseling by pharmacists improved compliance, reduced mortality, and reduced the use of healthcare resources in patients receiving polypharmacy [32]. Pharmacists may also encourage patients to discuss any problems with their healthcare teams; this might have influenced the drug regimens that they were prescribed, which resulted in better compliance and tolerance in this group.

Pharmacists believed that they could improve their ability to provide pharmaceutical care if they had access to relevant, patient-specific clinical data. Interestingly, patients reported being very supportive, making this data available to their healthcare providers, including pharmacists. If this is the case, an obstacle that would need to be addressed is the pharmacists' relationships with physicians. Pharmacists expressed frustration with the current lack of direct access to physicians. If pharmacists had clinical data on patients, the need for direct access to physicians would likely increase, because pharmacists could more effectively monitor patients' health and implement more focused interventions that require coordination with physicians.

It is clear that the healthcare system is not currently conducive to the important role of pharmacists and must change for patients to benefit from pharmaceutical service. 


\section{Conclusion}

The role of pharmacists in detecting, predicting and preventing prescription errors is crucial. Pharmacists in the UAE need to improve their pharmaceutical care skills in order to participate in the process of patient care, patient counseling and education. The dispensing role of the pharmacist has become obsolete. There is a need to improve pharmacist's knowledge in many aspects; pharmacological, medical and pharmacy practice. These aspects are necessary to perform prescription screening for the purpose of preventing any potential errors and detecting already existing ones.

\section{REFERENCES}

[1] M. Everard, M. Lesko and C. Wiback, "New Tool to Enhance Role of Pharmacists in Health Care," WHO, 2006.

http://www.who.int/mediacentre/news/new/2006/nw05/en/

[2] H. M. Belknap and S. A. Lanzotti, "Application of Software Design Principles and Debugging Methods to an Analgesia Prescription Reduces Risk of Severe Injury from Medical Use of Opioids," Clinical Pharmacology \& Therapeutics, Vol. 84, No. 3, 2008, pp. 385-392. http://www.ncbi.nlm.nih.gov/pubmed/18388884

[3] J. Netzer and K. C. Clauson, "Comparison of State Requirements for Preprinted Prescriptions,” American Journal of Health-System Pharmacy, Vol. 26, No. 21, 2005, pp. 2278-2282. http://www.medscape.com/viewarticle/515546_1

[4] M. F. Zwarenstein, K. N. Dainty and S. Quan, “A Cluster Randomized Trial Evaluating Electronic Prescribing in an Ambulatory Care Setting," Trials, Vol. 3, No. 8, 2007, p. 28. http://www.ncbi.nlm.nih.gov/pubmed/17915028

[5] A. R. Kuyper, "Patient Counseling Detects Prescription Errors,” Hospital Pharmacy, Vol. 28, No. 12, 1993, pp. 1180-1184. http://www.ncbi.nlm.nih.gov/pubmed/10130615

[6] F. A. Elizabeth, K. N. Barker and B. J. Carnahan, "National Observational Study of Prescription Dispensing Accuracy and Safety in 50," Journal of American Pharmacists Association, Vol. 43, No. 2, 2003, pp. 191-200. http://www.medscape.com/viewarticle/451962_print

[7] R. G. Neville, F. Robertson, S. Livingstone and I. K. Crombie, “A Classification of Prescription Errors," Journal of the Royal College of General Practitioners, Vol. 39, No. 320, 1989, pp. 110-112.

http://www.pubmedcentral.nih.gov/pagerender.fcgi?artid $=1711802 \&$ pageindex $=1$

[8] P. J. Barry, P. Gallagher, C. Ryan and D. O'mahony, "START (Screening Tool to Alert Doctors to the Right Treatment)-An Evidence-Based Screening Tool to Detect Prescribing Omissions in Elderly Patients,” Age Ageing, Vol. 36, No. 6, 2007, pp. 632-638. http://ageing.oxfordjournals.org/cgi/content/abstract/afm1 $\underline{18 \mathrm{v} 1}$

[9] B. Dean, M. Schachter and C. Vincent, "Prescribing Er- rors in Hospital Inpatients: Their Incidence and Clinical Significance,” Quality \& Safety in Health Care, Vol. 11, 2002, pp. 340-344.

http://qshc.bmj.com/cgi/content/abstract/11/4/340 http://dx.doi.org/10.1136/qhc.11.4.340

[10] M. G. Abdullah and N. Barber, "Systematic Review of Medication Errors in Pediatric Patients," Annals of Pharmacotherapy, Vol. 40, No. 10, 2006, pp. 1766-1776. http://www.medscape.com/viewarticle/549356

[11] P. P. Dobesh, "Managing Hypertension in Patients with Type 2 Diabetes Mellitus," American Journal of HealthSystem Pharmacy, Vol. 63, No. 12, 2006, pp. 1140-1149. http://www.medscape.com/viewarticle/535775

[12] B. Berenguer, C. La Casa, M. J. de la Matta and M. J. Calero, "Pharmaceutical Care: Past, Present and Future," Current Pharmaceutical Design, Vol. 10, No. 31, 2004, pp. 3931-3946.

http://www.ingentaconnect.com/content/ben/cpd/2004/00 $\underline{000010 / 00000031 / a r t 00009}$

[13] B. Henk, A. G. M. Peter, D. Leufkens, G. M. Hubert and E. C. G. Antoine, "Evaluation of the Clinical Value of Pharmacists’ Modifications of Prescription Errors,” British Journal of Clinical Pharmacology, Vol. 58, No. 5, 2004, pp. 503-511.

http://www.pubmedcentral.nih.gov/articlerender.fcgi?arti $\underline{\mathrm{d}=1884615}$

[14] C. J. Hawkey, S. Hodgson, A. Norman and T. K. Daneshmend, "Effect of Reactive Pharmacy Intervention on Quality of Hospital Prescribing,” BMJ, Vol. 300, 1990, pp. 986-990.

http://www.bmj.com/cgi/content/abstract/300/6730/986 http://dx.doi.org/10.1136/bmj.300.6730.986

[15] J. Y. Wick and G. R. Zanni, "Informed Consent: What Every Pharmacist Should Know,” Journal of American Pharmacists Association, Vol. 41, No. 4, 2001. http://www.medscape.com/viewarticle/406721

[16] M. R. Amsler, "Pharmaceutical Care in Chain Pharmacies: Beliefs and Attitudes of Pharmacists and Patients," Journal of American Pharmacists Association, Vol. 41, No. 6, 2002, pp. 850-855.

[17] W. Hammond, "Collaborative Drug Therapy Management by Pharmacists,” Pharmacotherapy, Vol. 23, No. 9, 2003, pp. 1210-1225.

http://www.medscape.com/viewarticle/461410 http://dx.doi.org/10.1592/phco.23.1.9.31916

[18] W. R. Vincent, K. M. Smith and D. Steinke, "Opportunities for Pharmacists in Public Health,” American Journal of Health-System Pharmacy, Vol. 64, No. 19, 2007, pp. 2002-2007.

[19] S. S. Sen and J. Thomas III, "Assessment of a PatientBased Pharmaceutical Care Scale," American Journal of Health-System Pharmacy, Vol. 57, No. 17, 2000. http://www.medscape.com/viewarticle/406909

[20] W. Nilaward, H. L. Mason and G. D. Newton, "Community Pharmacist-Child Medication Communication: Magnitude, Influences and Content," Journal of American Pharmacists Association, Vol. 45, 2005, pp. 354-362. http://dx.doi.org/10.1331/1544345054003778

[21] S. Kripalani, V. Vigil and K. R. Epstein, "Frequency and 
Predictors of Prescription-Related Issues after Hospital Discharge,” Journal of Hospital Medicine, Vol. 3, No. 1, 2008, pp. 12-19.

http://www.medscape.com/viewarticle/573055_4

[22] J. T. Lurk, "Effects of Changes in Patient Cost Sharing and Drug Sample Policies on Prescription Drug Costs and Utilization in a Safety-Net-Provider Setting," American Journal of Health-System Pharmacy, Vol. 61, No. 3, 2004, pp. 267-272.

http://www.medscape.com/viewarticle/468784_2

[23] C. W. Cranor and D. B. Christensen, "The Asheville Project: Short-Term Outcomes of a Community Pharmacy Diabetes Care Program," Journal of the American Pharmaceutical Association, Vol. 43, No. 2, 2003, pp. 149159.

http://www.ncbi.nlm.nih.gov/pubmed?term=12688433

[24] S. M. Gaffney, "Key Articles and Guidelines in the Management of Hypertension,” Pharmacotherapy, Vol. 28, No. 8, 2008, pp. 1041-1058. http://www.medscape.com/viewarticle/578718

[25] P. A. Howard, "Drug Therapy Recommendations from the 2005 ACC/AHA Guidelines for Treatment of Chronic Heart Failure,” The Annals of Pharmacotherapy, Vol. 40, No. 9, 2006, pp. 1607-1617.

[26] A. Marja, A. Riitta and E. Hannes, "The 'Questions to Ask about Your Medicines' Campaign: An Evaluation of Pharmacists and the Public's Response,” Medical Care, Vol. 36, No. 3, 1998, pp. 422-427.

[27] O. E. Boris and Anthonius, "Effectiveness of Interventions by Community Pharmacists to Improve Patient Adherence to Chronic Medication; a Systemic Review,” The annals of Pharmacotherapy, Vol. 39, 2005, p. 324.
[28] K. Beaucage, “Telephone Follow-Up of Patients Receiving Antibiotic Prescriptions from Community Pharmacies,” American Journal of Health-System Pharmacy, Vol. 63, No. 6, 2006, pp. 557-563.

http://www.medscape.com/viewarticle/527440_4

[29] R. L. Brown, "Pharmacoepidemiology of Potential Alcohol-Prescription Drug Interactions among Primary Care Patients with Alcohol-use Disorders," Journal of the American Pharmacists Association, Vol. 47, No. 2, 2007, pp. 135-139. http://www.medscape.com/viewarticle/557157

[30] L. Richwine, "U.S. FDA Proposes New Pregnancy Labels for Medicines,” Reuters Health Information, 2008. http://www.reuters.com/article/2008/05/28/us-drugs-preg nancy-idUSN2844317120080528

[31] L. A. Dent, J. G. Scott and E. Lewis, "Pharmacists-Managed Tobacco Cessation Program in Veterans Health Administration Community Based Outpatient Clinic," Journal of the American Pharmacists Association, Vol. 44, No. 6, 2005, pp. 700-715.

http://www.medscape.com/viewarticle/498148

[32] J. Y. F. Wu, W. Y. S. Leung, S. Chang, B. Lee, P. C. Y. Tong and J. C. N. Chan, "Effectiveness of Telephone Counseling by a Pharmacist in Reducing Mortality in Patients Receiving Polypharmacy: Randomized Controlled Trial,” BMJ, Vol. 333, 2006, p. 522. http://www.bmj.com/cgi/content/abstract/333/7567/522? maxtoshow $=\&$ HITS $=10 \&$ hits $=10 \&$ RESULTFORMAT $=\&$ fullte $\underline{\mathrm{xt}=\text { telephone\&searchid }=1 \& \text { FIRSTINDEX }=0 \text { \&volume }=3}$ 33\&issue $=7567 \&$ resourcetype $=$ HWCIT http://dx.doi.org/10.1136/bmj.38905.447118.2F 